PROCEEDINGS OF THE

AMERICAN MATHEMATICAL SOCIETY

Volume 127, Number 4, April 1999, Pages 963-965

S 0002-9939(99)04747-4

\title{
EQUATIONS IN FREE GROUPS ARE NOT FINITELY APPROXIMABLE
}

\author{
THIERRY COULBOIS AND ANATOLE KHELIF
}

(Communicated by Ronald M. Solomon)

\begin{abstract}
We give an equation in any free group $F$ of rank $\geq 2$ that has a solution in any finite quotient of $F$, but has no solution in $F$.
\end{abstract}

\section{INTRODUCTION AND RESULTS}

For all this paper we fix a non-commutative free group $F$. An equation is an identity of the form $w\left(x_{1}, \ldots, x_{n}, a_{1}, \ldots, a_{m}\right)=1$ where $x_{1}, \ldots, x_{n}$ are variables, $a_{1}, \ldots, a_{m}$ free generators of $F$ and $w$ a word in the free group on $x_{1}, \ldots, x_{n}$, $a_{1}, \ldots, a_{m}$. A solution to this equation is a tuple $g_{1}, \ldots, g_{n} \in F$ such that $w\left(g_{1}, \ldots, g_{n}, a_{1}, \ldots, a_{m}\right)=1$. In 1983 G. S. Makanin [4] described an algorithm to solve equations in free groups. G. Sabbagh asked the following question: does an equation in $F$ have a solution if and only if it has a solution in any finite quotient of $F$ ? Baumslag [1] proved that for an equation of the form $x^{-1} u x=v$ or $x^{p}=u$ $(u, v \in F, x$ a variable, $p$ a prime) the answer is yes. Recently the second author [3] proved that an equation of the form $x^{n}=u$ or $[x, y]=v$ (where $x, y$ are variables, $n$ an integer, $[x, y]=x^{-1} y^{-1} x y$ and $u, v$ are elements of $F$ ) has a solution in $F$ if and only if it has a solution in each finite quotient. We will say that these equations are finitely approximable. A positive answer to the question of G. Sabbagh would have yielded an alternative proof of Makanin's result.

Then, the second author proved, using some model theory, that there exists a finite system of equations that is not finitely approximable, and the first author, using the idea of the proof, gave the following equation that is not finitely approximable in $F$ :

$$
\left[x^{2} a, y^{-1} z^{2} b y\right]=t^{3}
$$

where $a, b$ are distinct members of a set of free generators of $F$ and $x, y, z, t$ are variables. We will give below a straightforward, purely algebraic proof of this fact, and we will leave it to the reader to similarly deal with the equation $\left[x^{p} a, y^{-1} z^{p} b y\right]=$ $t^{q}$ where $p$ and $q$ are two distinct primes.

\section{ProOF}

We first prove that (I) has a solution in any finite quotient of $F$. Let $G=F / N$ be a finite quotient of $F$. As any element of $G$ is of finite order, there exist two

Received by the editors July 15, 1997.

1991 Mathematics Subject Classification. Primary 20F10, 03B25, $20 \mathrm{E} 18$.

The authors wish to thank G. Sabbagh for suggesting this question to them. 
integers $\alpha$ and $\beta$ such that $a^{2 \alpha+1}$ and $b^{2 \beta+1}$ are elements of order a power of 2 . By Sylow's theorem there exists an element $y \in G$, such $a^{2 \alpha+1}$ and $y^{-1} b^{2 \beta+1} y$ generate a 2-subgroup of $G$. Now the commutator of these two elements is itself in this 2 -subgroup and is therefore a cube. Setting $x=a^{\alpha}$ and $z=b^{\beta}$ we conclude that (I) has a solution in $G$.

Now we prove that (I) has no solution in $F$. By a result of Schützenberger [6], a commutator is a proper power in a free group if and only if it is trivial. Therefore if (I) has a solution in $F$, then so does

$$
\left[x^{2} a, y^{-1} z^{2} b y\right]=1 .
$$

Consider the homomorphism $\varphi$ from $F$ onto $\mathrm{UT}_{3}(\mathbb{Z} / 2 \mathbb{Z})$ such that

$$
\varphi(a)=\left(\begin{array}{lll}
1 & 1 & 0 \\
0 & 1 & 0 \\
0 & 0 & 1
\end{array}\right) \text { and } \varphi(b)=\left(\begin{array}{lll}
1 & 0 & 0 \\
0 & 1 & 1 \\
0 & 0 & 1
\end{array}\right) .
$$

Then an easy calculation shows that for any $x, y, z \in F$ one has

$$
\varphi\left(\left[x^{2} a, y^{-1} z^{2} b y\right]\right)=\left(\begin{array}{ccc}
1 & 0 & 1 \\
0 & 1 & 0 \\
0 & 0 & 1
\end{array}\right) \neq 1 .
$$

This proves that equations (II) and (I) have no solution in $F$.

\section{Further REMARKS}

We would like to conclude with stressing the fact, presumably familiar to the experts, that the finite approximability of equations in free groups is linked to an outstanding problem. We have in mind the following conjecture :

Conjecture 1. In every (topologically) finitely generated profinite group, every subgroup of finite index is open.

B. Hartley [2] proved this conjecture for finitely generated pro-(finite and $\mathfrak{N}^{l}$ ) groups, where $\mathfrak{N}$ is the class of nilpotent groups. It is linked with the finite approximability of equations via the following conjecture and proposition :

Conjecture 2. For every integers $k, n$ there exists $f(n, k)$ such that in any finite group on less than $k$ generators any product of $n$-th power is a product of $f(n, k)$ $n$-th powers.

It is well known that in a non-Abelian free group the number of $n$-th powers necessary in such a product is not bounded. This conjecture is equivalent to the existence of $s$ such that all the equations of the form $x_{1}{ }^{n} \cdots x_{s}{ }^{n}=u$, where $u$ is a product of strictly more than $s n$-th powers in a free group on $k$ generators, are not finitely approximable. C. Martinez [5] proved that such a bound $f(n, k)$ exists for nilpotent groups.

Proposition. Conjecture 1 is a consequence of Conjecture 2.

Proof. Assume Conjecture 2, and let $G$ be a profinite group on $k$ generators. Let $H$ be a subgroup of $G$ of finite index. $H$ contains a normal subgroup $N$ of $G$ of finite 
index . Let $n$ be the index of $N$ in $G$. Let $\left\langle G^{n}\right\rangle$ be the subgroup of $G$ generated by the set $G^{n}$ of $n$-th powers in $G$. $\left\langle G^{n}\right\rangle$ is a subgroup of $H$. By Conjecture 2

$$
\left\langle G^{n}\right\rangle=\underbrace{G^{n} \cdot G^{n} \cdots G^{n}}_{f(n, k)}
$$

$G^{n}$ is compact and therefore $\left\langle G^{n}\right\rangle$ is a closed subgroup of $G . G /\left\langle G^{n}\right\rangle$ is a profinite group of bounded exponent and (topologically) finitely generated. By the positive solution of the restricted Burnside problem [7], it is a finite group and thus $\left\langle G^{n}\right\rangle$ is an open subgroup of $G$. We proved that $H$ is open in $G$.

For $n=2,3,4$, if every subgroup of index $n$ of a profinite group on $k$ generators is open, then $f(n, k)$ exists. Indeed let $G$ be a free profinite group on $k$ generators. Any group of exponent $n$ is residually finite, and thus $\left\langle G^{n}\right\rangle$ is closed in $G$. Using Lemma 1 of [2] we conclude that $f(n, k)$ exists.

\section{REFERENCES}

1. G. Baumslag, Residual nilpotency and relations in free groups, J. of Algebra 2 (1965) 271-282. MR 31:3487

2. B. Hartley, Subgroups of finite index in profinite groups, Mathematische Zeitschrift 168 (1979) 71-76. MR 80k:20028

3. A. Khelif, Some properties of free groups and their profinite completions, in preparation.

4. G.S. Makanin, Equations in a free group, Math. USSR Izv. 21 (1983) 483-546. MR 84m:20040

5. C. Martinez, On power subgroups of profinite groups, Trans. of the A. M. S. 345 (1994) 865-869. MR 95d:20053

6. M.P. Schützenberger, Sur l'equation $a^{2+n}=b^{2+m} c^{2+p}$ dans un groupe libre, Comptes Rend. de l'Ac. des Sciences (Paris) 248 (1959) 2435-2436. MR 21:2000

7. M.R. Vaughan-Lee, The restricted Burnside problem, London Math. Soc., Oxford 1990. MR 92c:20001

Equipe de Logique, UfR de Mathematiques, Universite Paris 7, 2 Place Jussieu, 75251 Paris Cedex 05, France 